\title{
Some Video Abstraction Techniques for Displaying Body Movement in Analysis and Performance
}

\section{Alexander Refsum Jensenius}

\section{$\mathrm{V}$}

isualization of human body movement has been a challenge for artists and researchers for centuries. The increasing interest in body movement among music researchers [1] has led to the need for simple and efficient techniques for recording, analyzing and presenting such movements in various ways. Since movements happen in space over time, they are not directly representable in two-dimensional displays on paper or screen. A key challenge then is to create displays that can effectively represent both temporal and spatial aspects of movement sequences.

In relation to our perceptual apparatus, it may be useful to differentiate between three temporal levels [2]:

- Sub-chunk level: perception of continuous sound and movement features

- Chunk level: fragments of sound and movement perceived holistically, i.e. sound objects and goal-directed actions, typically $0.5-5.0$ seconds

- Supra-chunk level: several chunks concatenated into larger structures

The aim of this paper is to give an overview of some videobased techniques for visualizing body movement at the three temporal levels and to show some examples of their use in scientific analysis and artistic practice.

\section{EARly Photographic Techniques AND MOTION CAPTURE}

\section{Early Photographic Techniques}

British-American photographer Eadweard James Muybridge (1830-1904) pioneered early movement analysis in California with pictures of the motion of animals and people [3]. Of his many interesting studies, he is best known for a series of time-lapse photographs of horses in the late 1870s, in which he effectively demonstrated that at certain moments in time a galloping horse has no legs on the ground [4]. Each recorded frame was captured when the horse stepped on a wire connected to a series of aligned cameras. Later Muybridge invented a clockwork mechanism to activate the cameras automatically [5]; he used this device to shoot an image sequence

Alexander Refsum Jensenius (researcher), University of Oslo, PB 1017 Blindern, 0315 Oslo, Norway. E-mail: <a.r.jensenius@imv.uio.no>.

See <www.mitpressjournals.org/toc/leon/46/1> for supplemental files associated with this issue. of a woman walking down steps (Fig. 1). In Muybridge's display, it is possible to get an impression of the subject's movement even though the time-lapse photograph is only a series of still pictures.

At around the same time as Muybridge's California experiments, Étienne-Jules Marey (also 18301904) was directing another type of photographic exploration in Paris [6]. He developed various types of what he called chronophotography or

"pictures of time." One such technique, strobophotography, was based on creating multiple exposures of a moving subject on the same photographic plate. An example of strobophotography is the walking "stick man" in Fig. 2. This photograph was taken with multiple exposures of a walking subject wearing a black suit with small reflective markers attached to the joints [7]. Here it is possible to get an idea of both the temporal and the spatial characteristics of the movement from a single picture.

In the 1970s, almost 100 years after Marey's experimentation with strobophotography, Swedish psychologist Gunnar Johansson used a similar technique in his point light displays [8]. Johansson used reflective tape on the main joints of the body and a special video recording technique to capture only the markers. Based on these recordings he showed that people could efficiently recognize various movement features from point light displays alone.

\section{Motion Capture}

The point light display technique was later used in both psychological and behavioral research, although it is now more common to create the recordings using motion capture systems. A number of different motion capture systems exist [9], including sensor-based systems (e.g. based on accelerometers) and camera-based systems. In the latter group, infrared markerbased systems are often regarded as the state of the art due to their high spatial and temporal precision and accuracy. In such systems, multiple cameras and infrared light sources are placed around the recording space, with the cameras recording the infrared light reflected from markers on the body. The final result is a 3D display with a high level of both temporal and spatial detail.

An advantage of marker/sensor-based motion capture systems is that they allow for reliable tracking of the position (and sometimes orientation) of individual body joints. Further- 


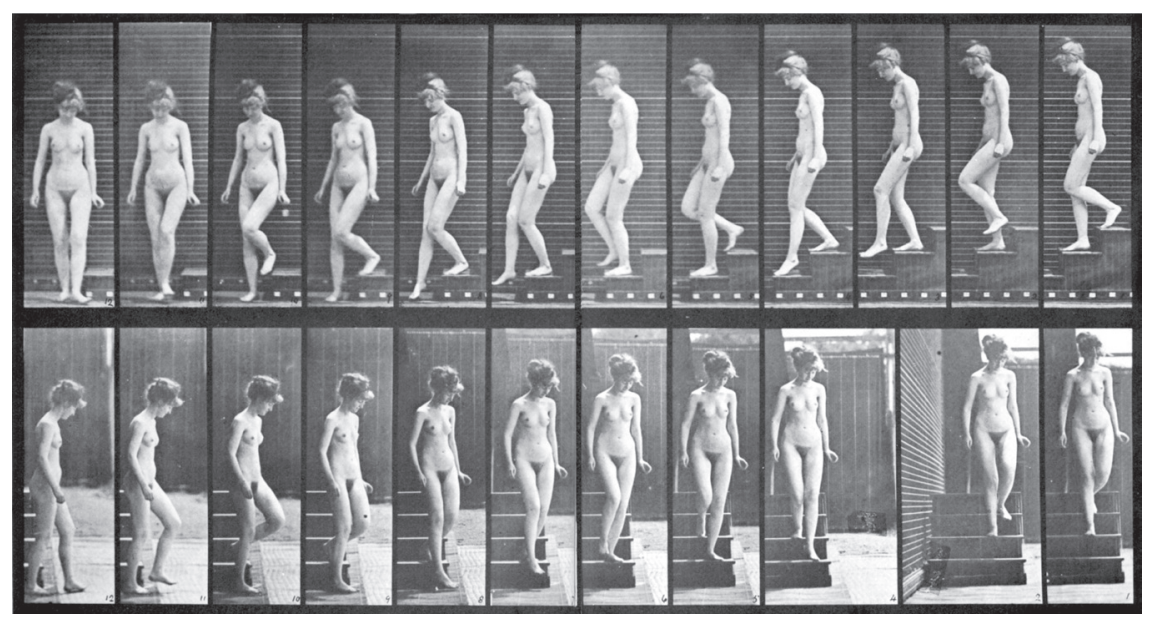

Fig. 1. Eadweard James Muybridge, Woman Walking Down Steps, photograph, 1887. This photograph is an example of a time-lapse photograph shot with a clockwork mechanism.

more, in combination with force plates and physiological sensors, such systems can provide very precise and accurate data about the kinematics and kinetics of human motion.

However, there are drawbacks to motion capture systems. Their price is one, although they have become somewhat more affordable in recent years. More problematic from a user's perspective are the constraints enforced by the systems. Infrared systems typically require extensive equipment and need to be installed in a controlled environment with few visual reflections. Sensor-based systems do not have such constraints but force the user to wear sensors and cables on the body. In my experience, the unnatural performance setting and equipment-oriented atmosphere that motion

(e.g. musicians and dancers) somew uncomfortable.

Recent advances in computer vision show that markerless 3D motion capture based on multi-camera video recordings may soon be a reality [10]. The development of cameras that can record the depth of objects in the image also makes it possible to use a single camera for markerless motion capture [11], as commercial systems such as Microsoft Kinect [12] have now begun to demonstrate. Such systems will undoubtedly open many new possibilities for both researchers and artists once further hardware and software solutions are available.

For many applications, however, we should not forget that regular video recording motion. The recent availability of better and cheaper video cameras makes them a good starting point for researchers, artists and students without access to motion capture systems. A challenge, however, has been the lack of basic techniques for visualizing movement from such video recordings. I have been exploring various visualization techniques suitable for the display of music-related movement, as well as certain easy-to-use applications that are freely available to the user community. These techniques and their applications are presented in the following sections.

\section{VIDEO-BASED VISUALIZATION TECHNIQUES}

The techniques presented here have been implemented in the graphical programming environment Max/MSP/ Jitter and are available both as source patches and as standalone applications [13]. cameras remain a very good option for

\section{Motion History Images}

One of the most common techniques in motion analysis from video files is creation of a motion image. A motion image represents the motion happening between two frames but does not represent a motion sequence happening over additional frames (i.e. the chunk level). Therefore it is necessary to create displays visualizing the motion itself over timeor what I refer to here as a motion history image. This problem has been explored previously through several different techniques - for example, motion-energy images [14], timed motion-history images [15] and silhouette motion images [16].

Fig. 2. Étienne-Jules Marey, Walking Man, chronophotography, 1884. This was based on adding reflective markers to the body of a person walking.

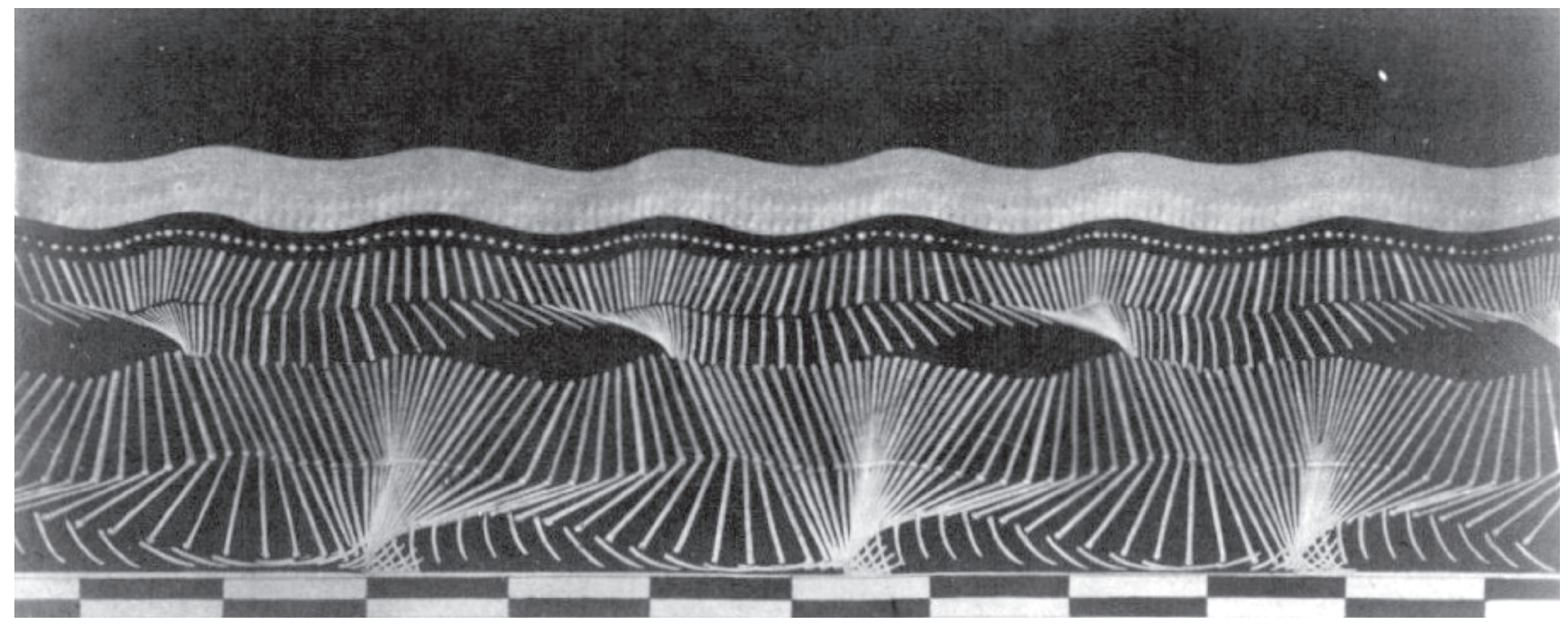




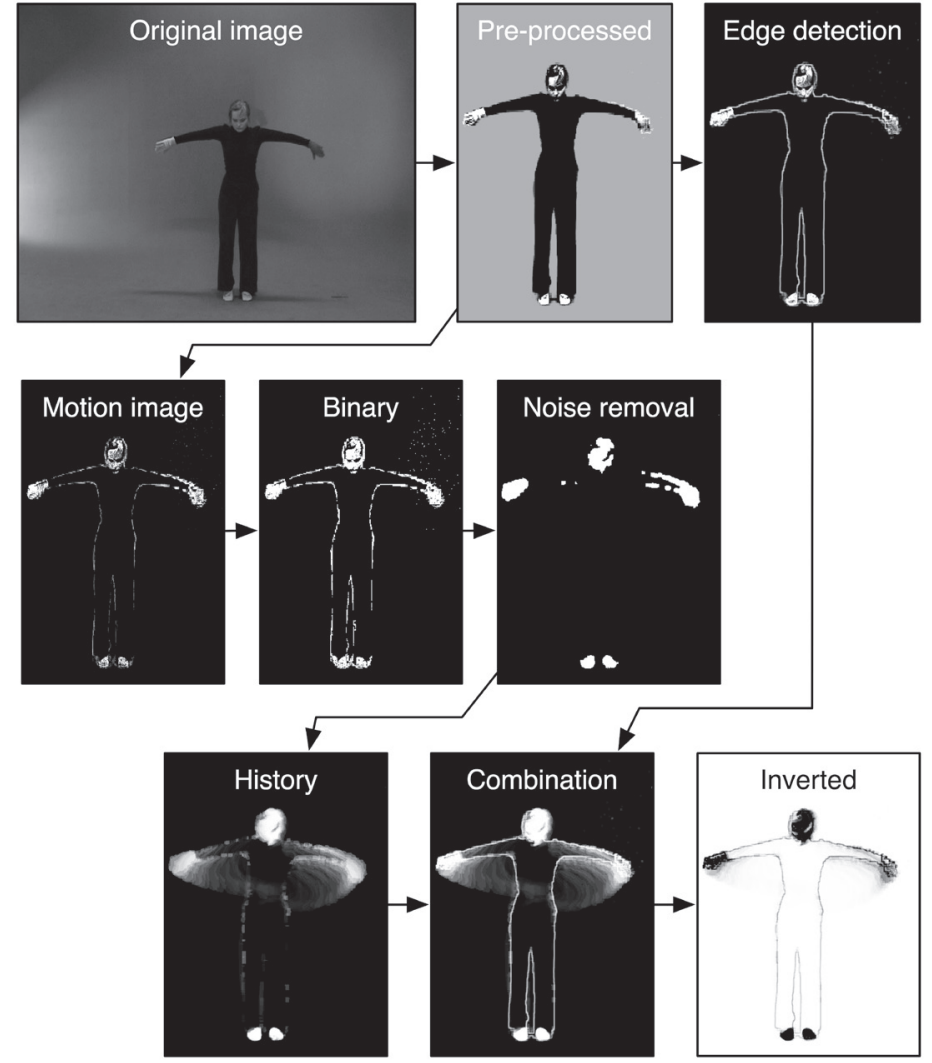

Fig. 3. An overview of the process of creating a motion history image. (@ A.R. Jensenius)
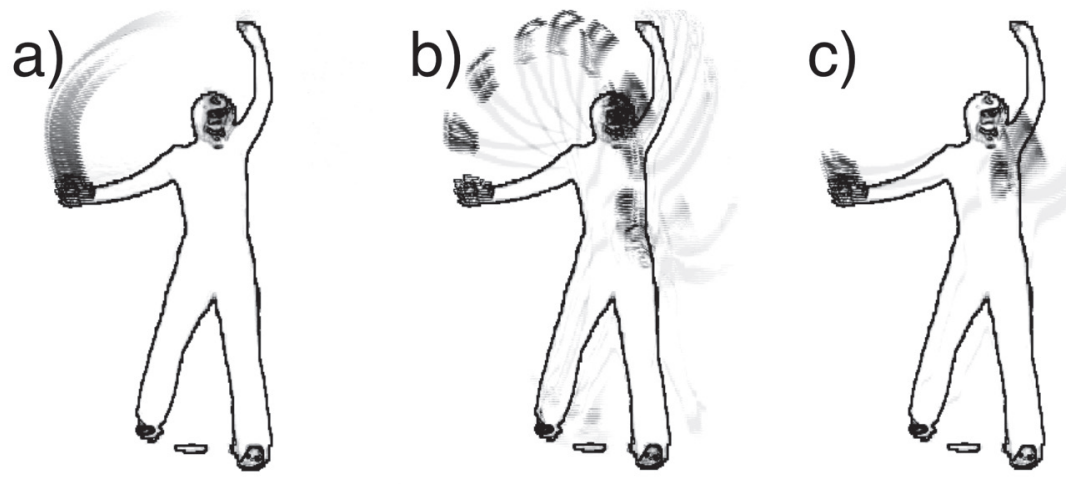

Fig. 4. Motion history images based on three different feedback techniques: (a) cell-wise temporal envelope follower; (b) multi-frame delay buffer; (c) "waterfall"-type delay. (๔ A.R. Jensenius)

Fig. 5. Three different durations of the motion history: (a) 3 frames; (b) 10 frames; (c) 30 frames. (@ A.R. Jensenius)
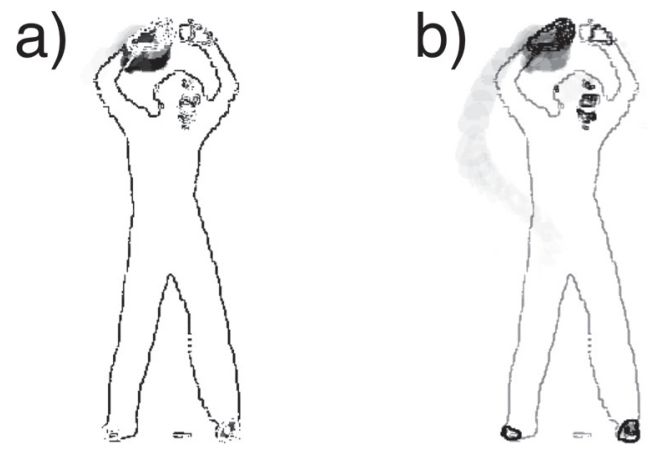

My approach to creating motion history images is shown in the overview in Fig. 3. First the original image is preprocessed by conversion to grayscale, adjustment of brightness and cropping to the desired region. Then an edge detection algorithm, Sobel, is used to find the contour of the person in the image. The motion image is developed by calculating the absolute pixel difference between successive frames in the pre-processed video file. The end result is an image in which only the pixels that have changed between the frames are displayed. The quality of the raw motion image depends on the quality of the original video stream. Small changes in lighting, camera movement, compression artifacts, etc. may all influence the final image. Such visual "noise" can be removed by converting the motion image to a binary image (with only black or white pixels) and applying a low-pass filter to remove pixels below a certain threshold.

To create the final motion history image, the processed motion image is run through a temporal blurring filter, or video feedback algorithm, before adding the result of the edge detection algorithm. The motion history image may also be inverted so that it is easier to see the motion if it is printed on paper. Here the images are shown in grayscale, but they can also be processed in color [17]. Depending on the content of the original video, a color motion history image can more clearly reveal certain parts of the body, e.g. the hands.

The type of video feedback used is important for the visual result of the motion history image. Figure 4 shows examples of three different motion history images, each representing a 2 -second movement sequence. Here three different techniques have been used for creating the motion history: (a) a cell-wise temporal envelope follower, (b) a multi-frame delay buffer and (c) a "waterfall"-type delay. I generally prefer the first of these techniques, because it better displays the continuous movement, while the latter two show increasingly longer periods between the frames making up the history.

A challenge when creating motion history images is to find a suitable duration for the "history." Figure 5 shows examples of three different durations of motion histories: 3, 10 and 30 frames, respectively. If the history is too long, the image will often become blurred and difficult to interpret. This is particularly problematic if there is a great deal of movement happening in the sequence. Similarly, too brief a motion history may 


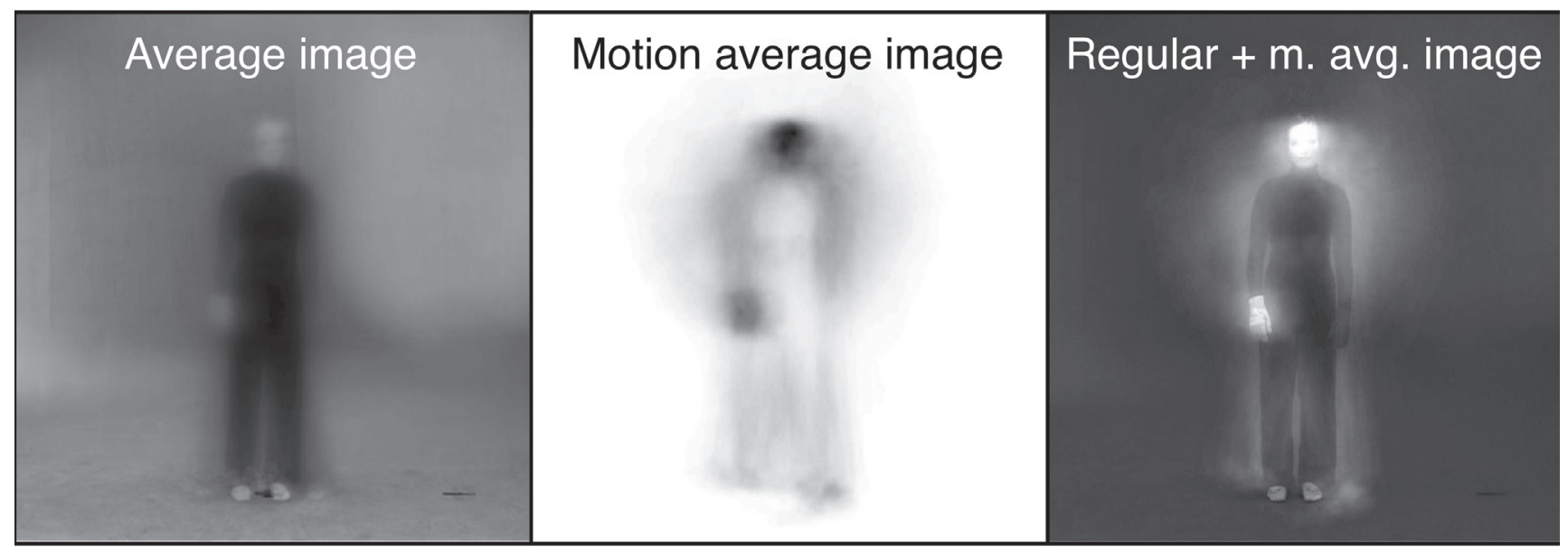

Fig. 6. Three different types of average images: (left) regular average image, (center) motion average image and (right) motion average image added to the first frame of the original video. These images display the spatial distribution of movement for the entire recording $(5 \mathrm{minutes})$. (@ A.R. Jensenius)

result in no visible effect if the movement in the sequence is slow.

\section{Motion History Keyframe Displays}

Motion history images may be used to represent movement at the chunk level, but rarely for more than 5 seconds. Extending the motion history beyond this will often result in images that are too blurred to be useful for analysis, although they may be interesting for creative applications. One solution for representing longer movement sequences is to create motion history keyframe displays. Keyframe displays similar to Muybridge's time-lapse photos (as seen in Fig. 1) have become the most common way of visualizing the content of video files in video editing programs. Although considerable experimentation has been done in creating more perceptually relevant keyframe displays [18], most such displays are still created by extracting still images from a video file at a regular interval.

When using motion history images as the basis for keyframe displays, we can more easily see the actual movement occurring over time, as shown in Color Plate G. Here each of the motion history images represents 2 seconds of video material, and thus the whole display represents 32 seconds of movement material. Even though this display is a crude reduction of the original material, it still presents quite a good impression of the movement sequences being performed by the dancer.

\section{Motion Average Images}

One way to visualize entire movement sequences (i.e. the supra-chunk level) is by calculating an average image over all frames in a video file. This approach can be seen as an analogue to an "open shutter" technique in photography, where all of the frames are combined to create a single image. The starting point for such a technique may be either the regular video or the motion image video, which will end up as average images or motion average images, respectively (Fig. 6). These images are based on a 5-minute video recording of stationary dance improvisation. The average images therefore give an impression of the spatial distribution of the entire recording.

Sometimes it may also be interesting to add the motion average image to a single frame from the original image. This al- lows representation in one display of both the sub-chunk and the supra-chunk level, since one can receive an impression of the scene and of the person in the scene while at the same time seeing the spatial distribution of the movement. Figure 7 shows such displays of a percussionist performing the same drumming pattern in 14 different ways; each sequence is about 15 seconds long.

\section{Motiongrams}

The motion average images reveal information about the spatial aspects of a motion sequence but do not show anything about the temporal development of the

Fig. 7. Motion average images overlaid the last frame of each video recording of a percussionist performing the same drumming pattern in 14 different ways. Each display represents around 15 seconds of video material. (@ A.R. Jensenius)
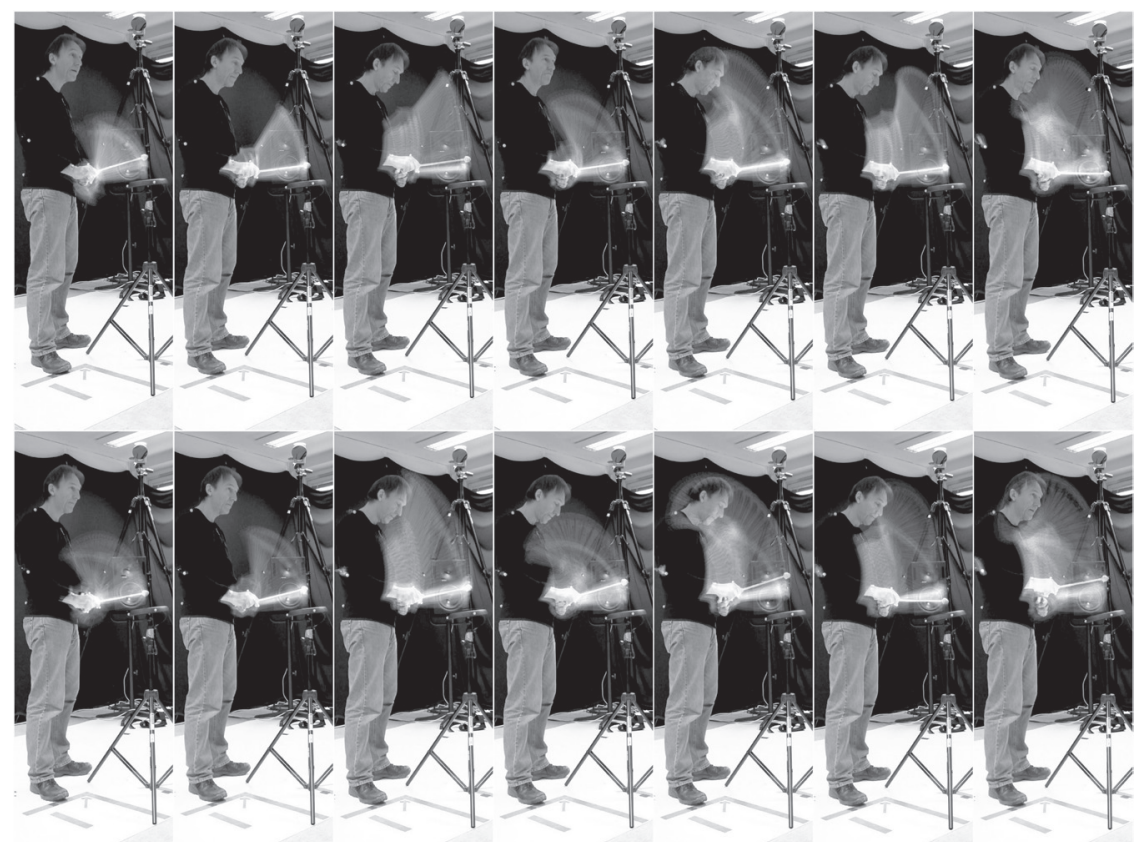


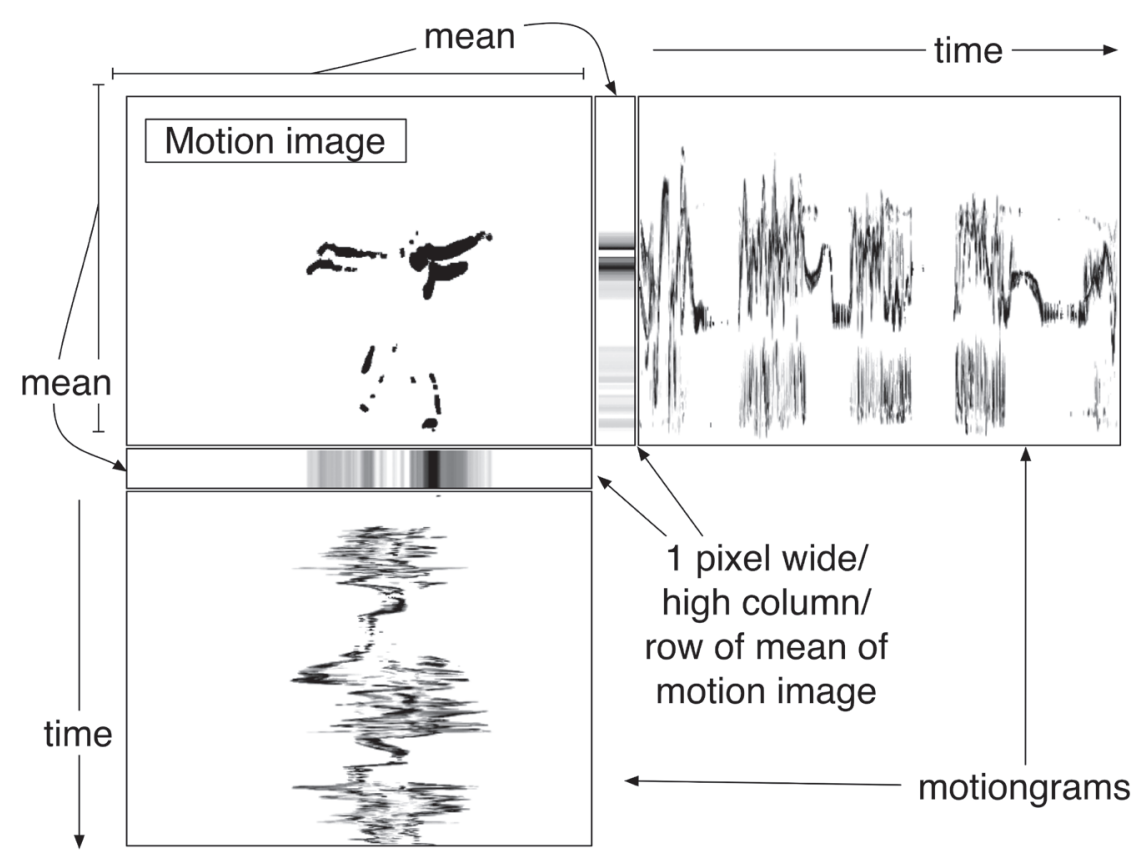

Fig. 8. An overview of the process of creating a motiongram, showing the motion image, the mean values of the motion images and the running motiongrams. (๑ A.R. Jensenius)

movements. Inspired by some of Marey's photographs and the long tradition of slit-scan photography [19], I started exploring displays of "collapsed" video frames. I call the resulting visual displays motiongrams. A motiongram resembles a slit-scan image, but with some differences. While slit-scan images are created by assembling single lines from subsequent frames, motiongrams are based on averaging over the whole image. Motiongrams are also calculated from the motion image, which make them somewhat different from video traces [20], displays based on silhouette images [21] or 3D motion "waterfall displays" [22].

An overview of the process of creating a motiongram is shown in Fig. 8. The technique is based on averaging over each of the frames in the input video, and drawing these 1-pixel-wide (or -tall) stripes next to one another. These running displays are motiongrams that make it possible to see both the location and the quantity of motion in a video sequence over time.

Figure 9 shows an example of a horizontal motiongram of the same dance sequence used in previous examples; the sequence displayed in Color Plate $\mathrm{G}$ is the same as in Section (a) 1-2 in Fig. 9. Here it is possible to identify the structure of the movement sequence from the motiongram. For example, it is possible to see that the dancer was mainly moving the upper regions of the body in the beginning (Section [a]) and moving the whole body more actively in the rest Jensenius) sock; these colors are very easily identifiable as yellow and red trajectories in a color motiongram [23].

It is important to remember that a motiongram is only a reduced display of a series of motion images. There is no analysis being done; the creation process is based only on a simple reduction algorithm. In a horizontal motiongram (e.g. Fig. 9) mainly vertical movement is visualized, because information about the spatial distribution of movement in the horizontal plane is only represented by 1 pixel for each row. Similarly, a vertical motiongram will mainly display the spatial distribution of horizontal movement. It might help to think of the motiongram as a display of a collapsed series of pictures, or "stripes," where each "stripe" summarizes the content of a whole motion image. When creating motiongrams, it is therefore necessary to evaluate in which plane(s) the movement is occurring before deciding whether to create a horizontal or a vertical motiongram (or both).

of the sequence. This motiongram also makes it easy to spot when the dancer was standing still, i.e. where there are few active pixels in the motiongram.

Due to the reduction that occurs when averaging over the rows (or columns) in the motion image, motiongrams cannot be used to identify individual limbs or body parts unless they are spatially separated. A solution may be to create motiongrams in color, with the averaging done for each of the four color planes in the motion image. The colors in the motiongram will then reflect the original colors in the video. In the recording used to generate Fig. 9, the dancer was wearing one yellow and one red glove and

\section{APPLICATIONS}

I and others have used the abovementioned movement visualization techniques in the analysis of various types of music-related movements, in medical applications and for creative uses.

\section{Performance Studies}

I have worked with others to use motiongrams and motion average images as visual "summaries" in several performance studies, e.g. of piano [24] and fiddle performances [25]. Here the images are often accompanied by spectrograms or score material, so that it is possible to study relationships between movement and sound. While such visualizations are

Fig. 9. Motiongram and motion average image of 5 minutes of dance improvisation to music, with time running from left to right. The dancer moved to 5 different musical excerpts (a-e), and each excerpt was repeated three times (1-3). A spectrogram of the musical sound makes it possible to look at relationships between sound and movement features. (๑ A.R.

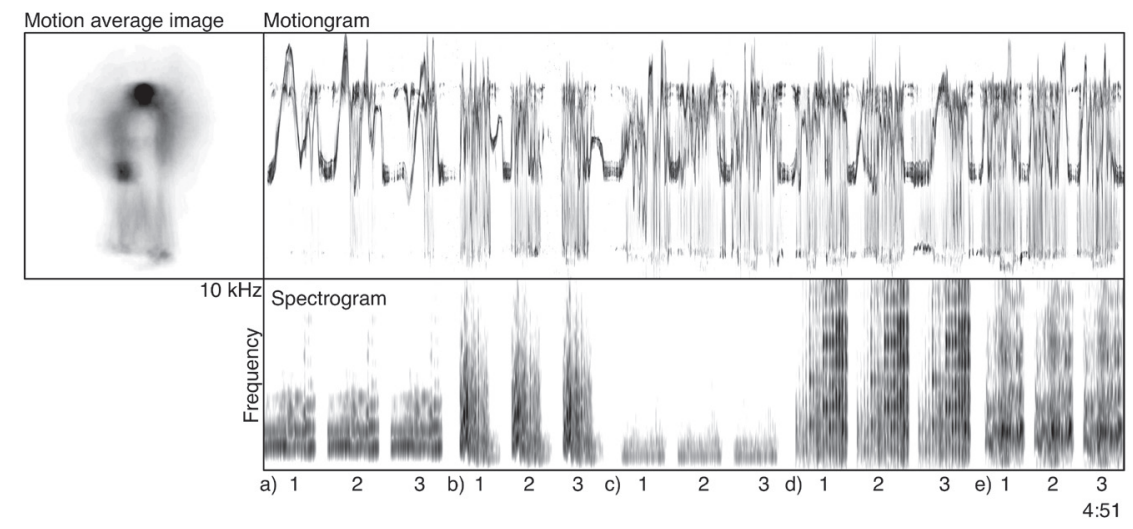




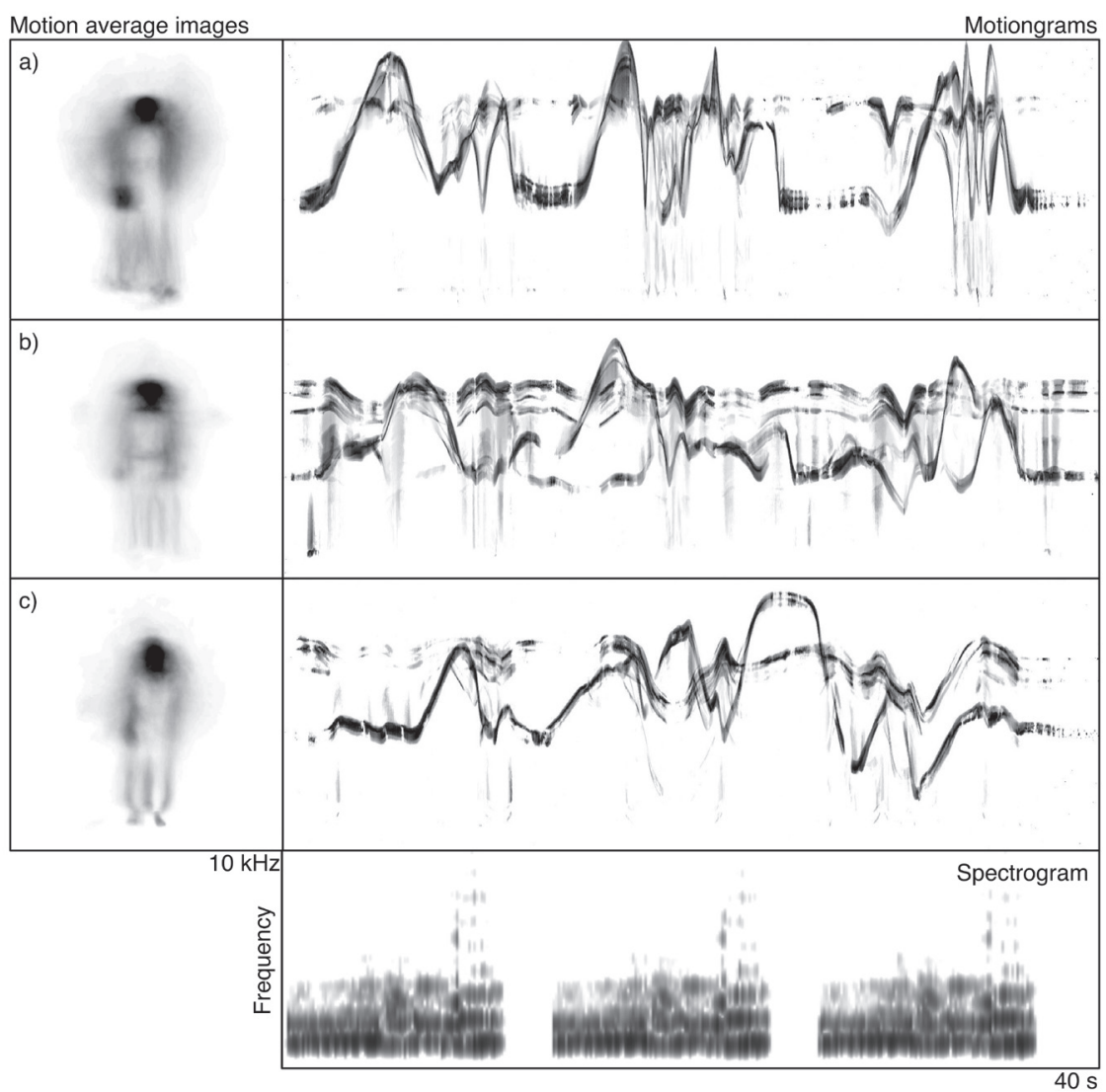

Fig. 10. These motion average images and motiongrams show the first 40 secs of recordings of three dancers improvising to the same musical material. (@ A.R. Jensenius)

useful for studying single recordings, I think they may be even more useful in comparative studies. Figure 10 shows motion average images and motiongrams of 40 seconds of three dance improvisations to the same musical material. Compared to the motiongram of the full dance sequence (Fig. 9), these motiongrams re-

veal more details and facilitate following the trajectories of the hands and heads of the dancers.

\section{Medical Applications}

While the visualization techniques presented above have been developed and explored mainly with music-related mate-

Fig. 11. These examples show average images and motiongrams of movement sequences of infants (a) with fidgety movements and (b) without fidgety movements. ( A.R. Jensenius)

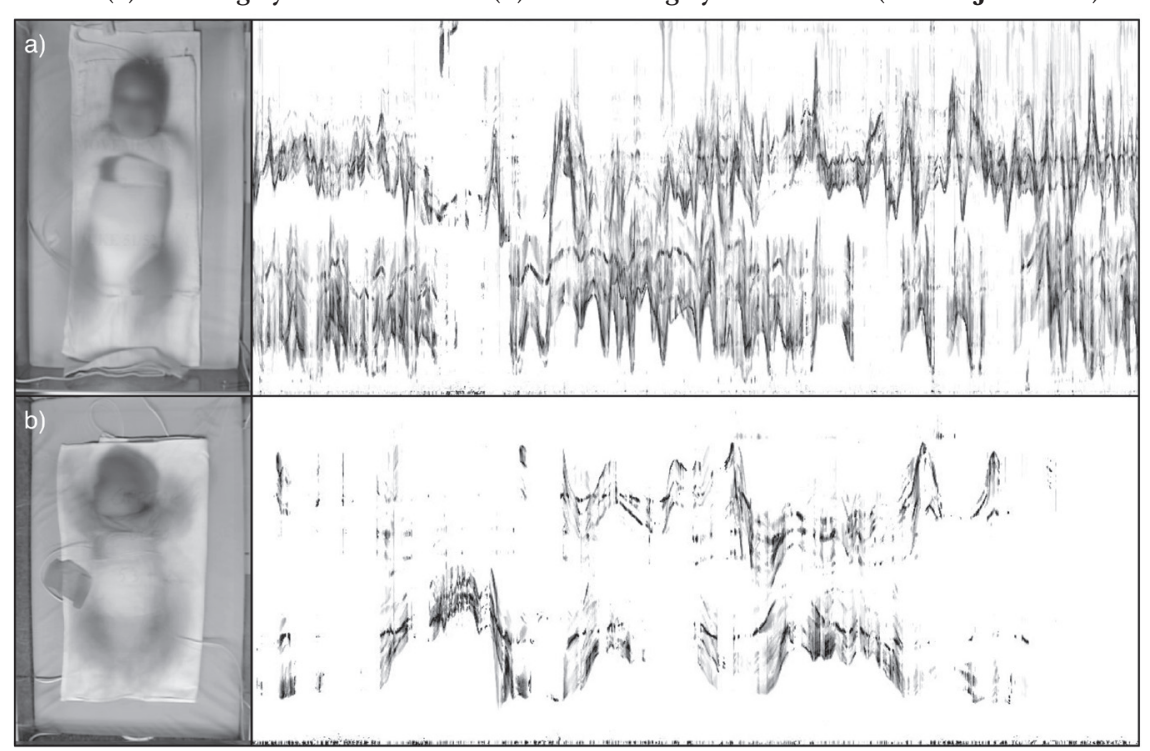

rial in mind, they have also been used in two different types of medical research. One of these is in animal experiments on attention deficit (hyperactivity) disorder (ADD and ADHD) [26]. Another is in the study of young infants at risk of developing cerebral palsy (CP) [27].

One way of detecting cerebral palsy at an early stage is by looking for the absence of so-called fidgety movements at 2-4 months. Developing easily used video analysis tools may be an important solution to screening more children and selecting those at risk of cerebral palsy for further evaluation by an expert clinician. Figure 11 shows how motiongrams clearly make it possible to identify the absence of fidgety movements.

\section{Artistic Use}

Besides the analytic use of the different visualization tools presented in this paper, I have also started exploring their potential in artistic use. In several concerts, I have projected combinations of motion images, average images and motiongrams behind the performers. Particularly in performances that involve computers, the movements of the performers are often less "expressive" than in other types of performance, thus I find that such abstract visuals work well. Future research will include further experimentation with such displays and interviews for feedback from performers and audiences.

Another use of these visualization techniques that I have explored is in sonification of body movement. By treating a motiongram as a spectrogram and running it through an inverse FFT process, it is possible to control the sound directly through movement [28]. Using motiongrams as the basis for sound generation makes for a very close link between movement and sound. Also, since sound is generated from image, it is possible to use video effects (e.g. brightness adjustments, feedback and blurring) as sound effects, since changing the video will also influence the generated sound. I will look into such relationships between movement and sound further in future research, including exploration of spacefilling curves in sonification [29].

\section{SUMMARY, DISCUSSION AND FUTURE WORK}

Inspired by early experiments in photography, as well as recent video abstraction techniques, I have explored and developed various video-based solutions for movement visualization: motion history images, motion average images, motion his- 
tory keyframe displays and motiongrams. Although the main focus has been on the creation of visual displays that can be printed on paper or shown statically on screen, all these techniques can also be used in real time.

The displays address different needs in representing body movement at different temporal levels. Motion history images may be used to visualize movement trajectories over short periods of time (up to around 5 seconds) or for longer sequences when combined into motion history keyframe displays. Motion average images can display the spatial distribution of entire recordings, but with no reference to temporal development. Motiongrams, on the other hand, can be used to display the spatiotemporal development of longer movement sequences-from a few minutes to several hours. Separately, or preferably together, these different displays are useful as movement summaries, for navigation and in comparative studies.

While the different visualization techniques presented here solve some problems, many unresolved issues remain:

- Large differences between rapid and slow movements are difficult to visualize. Particularly for motion history images, it is important to choose a duration of the feedback that is appropriate for the motion content. This necessarily involves a subjective evaluation of the material and judgment of the visual result in the creation process, as exemplified in Color Plate G. Motiongrams are better at visualizing large differences between fast and slow movements, as shown in Fig. 11, but here also a selection of the filtering is crucial for the visual result.

- Starting from a regular video recording (as opposed to multi-camera, infrared, depth-camera, etc.) necessarily imposes some limitations on the approach. Fortunately, humans are very good at imagining $3 \mathrm{D}$ motion by looking at a 2D display (e.g. photo or video). However, rotations of the body, for instance, are difficult to represent properly, although motion history keyframe displays (e.g. Color Plate G) may serve better for visualizing of such movements than a standard keyframe display. Motiongrams are even more reductionist, because they mainly visualize movement in one dimension. This could, however, also be argued to be one of the strengths of the approach, because a motiongram is able to display both the quantity and the location of motion in a compact display.

- The presented techniques are optimized for steady camera recordings. Because all the techniques begin from the motion image, any type of camera motion will greatly influence the visual result. Employing background subtraction and more advanced motion tracking techniques (e.g. optical flow) could improve the results. However, this would also increase the complexity of the implementations and the computational load.

- All the examples in this paper have been of a single subject. Motion history keyframe displays can easily be used to visualize the motion of several subjects at a time. Motiongrams, on the other hand, are somewhat more problematic because they are based on averaging over each video frame. If two or more people are standing next to one another, a horizontal motiongram will not make much sense for analytical applications, since it would display the combination of the movements. A vertical motiongram, however, could display the motion of each person in the frontal plane. For creative applications I have found that motiongrams of complex video material can be very interesting to work with, following in the tradition of slit-scanning [30].

While the aim of this work has been to create visualizations from regular video recordings, I am currently also exploring different visualization and sonification techniques based on 3D motion capture data [31].

Despite the limitations mentioned above, I have found that the different visualization techniques presented here and the accompanying easy-to-use computer programs are appealing to music researchers, students and artists. I have seen that researchers who would not be inclined to start working with more complex motion capture solutions have been able to create visualizations of their video material very quickly and easily. One of the strengths of these different techniques has been their wide application, ranging from analytical studies of dance and performance movements to medical applications. Also, their inspiration from, and use for, artistic applications attests to the close connections between scientific and artistic research and development.

\section{Acknowledgments}

Thanks to Rolf Inge Godøy and Marcelo M. Wanderley for many comments and suggestions on the early stages of this work, the Jamoma team for fruitful collaboration over many years, and to Åshild Ravndal Salthe, Kjell Samkopf and Lars Adde for providing material to the various illustrations in the paper.

\section{References}

Unedited references as provided by the author.

1. See overviews in e.g. Gritten, A. and E. King (eds.) (2006). Music and Gesture. Hampshire: Ashgate; Godøy, R.I. and M. Leman (Eds.) (2010). Musical Gestures: Sound, Movement, and Meaning. New York: Routledge.

2. Godøy, R.I. and A.R. Jensenius (2009). Body movement in music information retrieval. In Proceedings of the 10th International Society for Music Information Retrieval Conference, Kobe, Japan, pp. 45-50.

3. Clegg, B. (2007). The man who stopped time: The illuminating story of Eadweard Muybridge: Pioneer photographer, father of the motion picture, murderer. Washington, DC: Joseph Henry Press.

4. See animations based on some of Muybridge's photographs at <http://en.wikipedia.org/wiki/ Eadweard/Muybridge $>$.

5. Prodger, P. (2003). Time Stands Still: Muybridge and the Instantaneous Photography Movement. Oxford: Oxford University Press, p. 188

6. Braun, M. (1992). Picturing Time. The work of Etienne-Jules Marey (1830-1904). Chicago, IL: The University of Chicago Press.

7. Blake, R. and M. Shiffrar (2007). Perception of human motion. Annual Review of Psychology 58, 47-73.

8. Johansson, G. (1973). Visual perception of biological motion and a model for its analysis. Perception $\mathcal{E}^{\circ}$ Psychophysics 14(2), 201-211.

9. Skogstad, S.A., A.R. Jensenius, and K. Nymoen. Using IR optical marker based motion capture for exploring musical interaction. In Proceedings of New Interfaces for Musical Expression, Sydney, pp. 407-410.

10. Sigal, L., A. Balan, and M. Black. Humaneva: Synchronized video and motion capture dataset and baseline algorithm for evaluation of articulated human motion. International journal of computer vision, 87(1):4-27, 2010.

11. Pekelny, Y. and C. Gotsman (2008). Articulated object reconstruction and markerless motion capture from depth video. Computer Graphics Forum 27(2), 399-408.

12. <www.xbox.com/en-US/kinect >

13. The software is available from <www.fourms.uio. no>. Details on the implementation can be found in Jensenius, A.R. (2007). Action-Sound: Developing Methods and Tools to Study Music-Related Body Movement. Ph.D. thesis, University of Oslo. The video modules have been collected in the Musical Gestures Toolbox, which is now included in the Jamoma open framework <www.jamoma.org $>$.

14. Bobick, A.F. and J.W. Davis (2001). The recognition of human movement using temporal templates. IEEE Transactions on Pattern Analysis and Machine Intelligence 23(3), 257-267.

15. Bradski, G.R. and J.W. Davis (2002). Motion segmentation and pose recognition with motion history gradients. Machine Vision and Applications 13(3), 174-184.

16. Camurri, A., I. Lagerlöf and G. Volpe (2003). Recognizing emotion from dance movement: Comparison of spectator recognition and automated techniques. International Journal of Human-Computer Studies 59(1-2), 213-225.

17. Real-time color version of the technique: $<w w w$. youtube.com/watch?v=UPnEOT-5fgE $>$.

18. Lee, H., A. Smeaton, C. Berrut, N. Murphy, S Marlow and N. O'Connor (2000). Implementation and analysis of several keyframe-based browsing in- 
terfaces to digital video. In J. Borbinha and T. Baker (Eds.), Research and Advanced Technology for Digital Libraries, pp. 206-218. Springer Verlag.

19. Levin, G. (2005). An informal catalogue of slitscan video artworks: <www.flong.com/texts/lists/ slit_scan> (7 September 2010).

20. Nunes, M., S. Greenberg, S. Carpendale and C. Gutwin (2007). What did I miss? Visualizing the past through video traces. In ECSCW'07: Proceedings of the Tenth European Conference on Computer Supported Cooperative Work, pp. 1-20.

21. Liu, Y., R. Collins and Y. Tsin (2002). Gait sequence analysis using frieze patterns. In A. Heyden, G. Sparr, M. Nielsen, and P. Johansen (Eds.), Computer Vision-ECCV 2002, Volume 2351, pp. 733-736. Springer Berlin Heidelberg.

22. Hilpoltsteiner, M. (2005). Recreating movementTools for analyzing film sequences. Diploma thesis, University of Applied Sciences, Wuerzburg, Germany.

23. Examples of motiongrams in color: $<$ www.arj. no/2006/11/01/motiongrams/>

24. Godøy, R.I., A.R. Jensenius, and K. Nymoen (2010). Chunking in music by coarticulation. Acta Acoustica united with Acoustica 96(4), pp. 690-700.

25. Schoonderwaldt, E. and A.R. Jensenius (2011). Effective and expressive movements in a FrenchCanadian fiddler's performance. In Proceedings of the International Conference on New Interfaces for Musical Expression, Oslo, Norway, pp. 256-259.
26. Sagvolden, T., E.B. Johansen, H. Aase, and V.A. Russell (2005). A dynamic developmental theory of attention-deficit/hyperactivity disorder (ADHD) predominantly hyperactive/impulsive and combined subtypes. Behavioral and Brain Sciences 28(03), 397-419.

27. Adde, L., J.L. Helbostad, A.R. Jensenius, G Taraldsen, K. Grunewaldt, and R. Støen (2010). Early prediction of cerebral palsy by computerbased video analysis of general movements: A feasibility study. Developmental Medicine $\mathcal{E} \mathcal{F}^{2}$ Child Neurology 52(8), 773-778.

28. Sonification example: <www.youtube.com/ watch? $v=z z 5 S C P 5 Z k c U>$. More information in: Jensenius, A.R. (2012). Motion-sound interaction using sonification based on motiongrams. In Proceedings of ACHI 2012: The Fifth International Conference on Advances in Computer-Human Interactions, Valencia, Spain, pp. 170-175.

29. Grond, F. (2007). Organized data for organized sound. Space filling curves in sonification. In Proceedings of the 13th International Conference on Auditory Display, Montreal, pp. 476-482.

30. A recent example of such usage is Daniel Crooks's video art pieces in the series Static: $<$ http://daniel crooks.com/>.

31. Some recent examples of visualizations based on 3D motion capture can be seen in Shan, G., P. Visentin, and T. Harnett. A novel use of 3D motion capture: Creating conceptual links between technology and representation of human gesture in the visual arts. Leonardo, 43(1), 2010.

\section{Glossary}

average image - the mean value of all frames in a video stream.

motion average image - the mean value of all frames in a motion image video stream.

motion history image-shows trajectories of motion patterns based on applying feedback to a motion image.

motion history keyframe display-multiple motion history images presented in a grid.

motion image - the difference between two consecutive video frames.

motiongram-shows either horizontal or vertical motion based on drawing each motion image as a single line in the display.

Alexander Refsum Jensenius (BA, MA, MS, Ph.D.) is a music researcher and research musician working in the fields of embodied music cognition and new instruments for musical expression at the University of Oslo and Norwegian Academy of Music.

Manuscript received 4 March 2011.

\section{The Leonardo Vision}

Leonardo/ISAST is a non-profit organization that creates opportunities for the powerful exchange of ideas between practitioners in art, science and technology. Through publications, initiatives and public forums, Leonardo/ISAST facilitates cross-disciplinary research in these fields, seeking to catalyze fruitful solutions for the challenges of the 21st century. Among the challenges requiring cross-disciplinary approaches are establishing sustainable environmental practices, spreading global scientific and artistic literacy, creating technological equity, and encouraging freedom of thought and imagination. By enhancing communication between scientists, artists, and engineers, Leonardo supports experimental projects and interacts with established institutions of art and science to transform their research and educational practices.

As a 501(c) 3 non-profit organization, Leonardo depends on its community of supporters to fund its programs. These goals and actions are made possible by donors like you!

Donations to Leonardo are tax-deductible as provided by law. There are a number of ways to make a donation-please visit <www.leonardo.info/isast/donations.html > or contact us at Leonardo/ISAST, 211 Sutter Street, Suite 501, San Francisco, CA 94108, U.S.A. 